\title{
BioNERO: an all-in-one R/Bioconductor package for comprehensive and easy biological network reconstruction
}

Fabricio Almeida-Silva ${ }^{1 *}$ and Thiago M. Venancio ${ }^{1 *}$

${ }^{1}$ Laboratório de Química e Função de Proteínas e Peptídeos, Centro de Biociências e Biotecnologia, Universidade Estadual do Norte Fluminense Darcy Ribeiro, Campos dos Goytacazes, RJ, Brazil.

*FA-S: Laboratório de Química e Função de Proteínas e Peptídeos, Centro de Biociências e Biotecnologia, Universidade Estadual do Norte Fluminense Darcy Ribeiro. Av. Alberto Lamego 2000, P5, sala 217, Campos dos Goytacazes, RJ, Brazil. Email: fabricio almeidasilva@hotmail.com

*TMV: Laboratório de Química e Função de Proteínas e Peptídeos, Centro de Biociências e Biotecnologia, Universidade Estadual do Norte Fluminense Darcy Ribeiro. Av. Alberto Lamego 2000, P5, sala 217, Campos dos Goytacazes, RJ, Brazil. Email: thiago.venancio@gmail.com 


\section{ABSTRACT \\ 2 Summary}

3 Currently, standard network analysis workflows rely on many different packages, often

4 requiring users to have a solid statistics and programming background. Here, we present

5 BioNERO, an $\mathrm{R}$ package that aims to integrate all aspects of network analysis workflows, 6 including expression data preprocessing, gene coexpression and regulatory network 7 inference, functional analyses, and intra and interspecies network comparisons. The state-of8 the-art methods implemented in BioNERO ensure that users can perform all analyses with a 9 single package in a simple pipeline, without needing to learn a myriad of package-specific 10 syntaxes. BioNERO offers a user-friendly framework that can be easily incorporated in 11 systems biology pipelines.

12

\section{Availability and implementation}

14 The package is available at Bioconductor (http://bioconductor.org/packages/BioNERO). 


\section{Introduction}

18 To date, several packages have been developed to infer gene coexpression networks (GCNs) and gene regulatory networks (GRN) from expression data, such as WGCNA (Langfelder and Horvath, 2008), CEMiTool (Russo et al., 2018), petal (Petereit et al., 2016), and minet (Meyer et al., 2008). However, none of them can handle all aspects of network analysis workflows,

22 and users are required to use other packages to build a standard analysis pipeline. Further,

23 network inference requires a solid linear algebra and statistics background, resulting in a

24 struggle for inexperienced researchers to properly preprocess their expression data and

25 extract biologically meaningful information from the inferred networks.

Here, we present BioNERO (Biological Network Reconstruction Omnibus), an $\mathrm{R} /$ Bioconductor package that integrates all steps of network inference workflows in a single package. BioNERO uses state-of-the-art methods to preprocess expression data, infer GCNs and GRNs from expression data, analyze networks for biological interpretations, and compare networks within and across species. Additionally, BioNERO can be used to explore topological properties of protein-protein interaction networks, such as hub identification and community

32 detection.

\section{Implementation}

35 BioNERO is an R package that integrates existing functionalities and introduces new ones. The 36 input data can be common Bioconductor classes, such as SummarizedExperiment objects 37 (Morgan et al., 2020) for expression data, or basic R object classes, ensuring interoperability 38 with other packages. Long-running functions, such as that used for Fisher's exact tests in 39 overrepresentation analyses, have been parallelized with BiocParallel (Morgan et al., 2021) 40 to increase speed.

\subsection{Data preprocessing}

43 Networks inferred from unfiltered data often do not satisfy the scale-free topology (SFT) 44 assumption. Although this can be a property of the input data (particularly for heterogenous 45 data sets), this issue mainly results from a lack of systematic preprocessing. In BioNERO, 46 expression data are preprocessed prior to network inference to i. remove missing data; ii. 47 remove genes with low expression across samples; iii. select genes with the highest variances 48 (optional) and; iv. remove confounders that could introduce false-positive correlations. Count 
data can also be variance stabilizing transformed with DESeq2's algorithm (Love et al., 2014)

50 to make the expression matrix approximately homoscedastic. The resulting quantile 51 normalized expression data are adjusted for confounders based on a previously developed 52 principal component-based method (Parsana et al., 2019).

\section{$54 \quad 2.2$ Gene coexpression network inference}

55 We implemented the popular Weighted Gene Coexpression Network Analysis (WGCNA) 56 (Langfelder and Horvath, 2008) algorithm in BioNERO to infer weighted networks from

57 expression data. Users can infer three types of GCNs (signed, signed hybrid or unsigned), and

58 pairwise gene-gene correlations can be calculated with Pearson's r, Spearman's $\rho$, or biweight

59 midcorrelation (median-based, which is less sensible to outliers). Downstream GCN analyses

60 in BioNERO include module stability evaluation, hub gene identification, functional enrichment 61 analyses, subgraph extraction and network visualization. For all subgraph extractions, users 62 can verify if the graphs fit the SFT, which is characteristic of real-world biological networks 63 (Barabási et al., 2011). Additionally, BioNERO can be used to calculate main network 64 statistics, namely connectivity, scaled connectivity, clustering coefficient, maximum 65 adjacency ratio, density, centralization, heterogeneity, number of cliques, diameter, 66 betweenness, and closeness.

\subsection{Gene regulatory network inference}

69 Different GRN inference algorithms can be the best performers depending on the benchmark 70 expression data set, as demonstrated by Marbach et al. (2012). This observation inspired the 71 "wisdom of the crowds" principle for GRN inference, which consists in calculating average 72 ranks for all edges across different algorithms to obtain consensus, high-confidence edges 73 (Marbach et al., 2012). Here, we implemented three widely used GRN inference algorithms:

74 GENIE3 (Huynh-Thu et al., 2010), ARACNE (Margolin et al., 2006), and CLR (Faith et al.,

75 2007). However, choosing the most appropriate number of top edges to keep is a persisting 76 bottleneck, and users often pick an arbitrary number. We implemented a method to simulate 77 different networks by splitting the graph in $n$ subgraphs, each containing the top $n^{\text {th }}$ quantiles. 78 Then, we calculate SFT fit statistics for each subgraph and select the top number of edges 79 that leads to the best SFT fit. 


\subsection{Network comparison}

82 GCNs inferred from different expression sets have similarities and divergences. We

83 implemented two network comparison features in BioNERO, namely consensus module

84 identification and module preservation. Consensus modules are gene modules that co-occur

85 in networks inferred from independent expression sets, and they can be used to explore core

86 components of the studied phenotype that are not affected by experimental effects or natural

87 biological variation. While consensus modules identification focuses on the similarities

88 between networks, module preservation focuses on the differences, and it can be used to

89 explore patterns of transcriptional divergence within and across species. For interspecies

90 comparisons, BioNERO can interoperate with OrthoFinder (Emms and Kelly, 2015) to analyze

91 expression profiles at the orthogroup level.

92

\section{Benchmark}

94 A benchmark using maize (Zea mays) and rice (Oryza sativa) gene expression data obtained 95 from Shin et al. (2020) is available as Supplementary Text online.

96

\section{Conclusions}

98 BioNERO is a novel $\mathrm{R}$ package that integrates all steps of network analysis pipelines, providing 99 users with a simple framework for GCN and GRN inference from expression data. This package 100 can be easily integrated in systems biology pipelines and will likely accelerate biological 101 network analysis projects.

\section{Acknowledgements}

104 This work was supported by Fundação Carlos Chagas Filho de Amparo à Pesquisa do Estado 105 do Rio de Janeiro (FAPERJ; grants E-26/203.309/2016 and E-26/203.014/2018), 106 Coordenação de Aperfeiçoamento de Pessoal de Nível Superior - Brasil (CAPES; Finance Code 107 001), and Conselho Nacional de Desenvolvimento Científico e Tecnológico. The funding 108 agencies had no role in the design of the study and collection, analysis, and interpretation of 109 data and in writing.

110 Conflicts of interest: none declared. 


\section{REFERENCES}

Barabási,A.-L. et al. (2011) Hierarchical Organization of Modularity in Complex Networks. Science (80-. )., 297, 46-65.

Emms,D.M. and Kelly,S. (2015) OrthoFinder: solving fundamental biases in whole genome comparisons dramatically improves orthogroup inference accuracy. Genome Biol., 16, $1-14$.

Faith,J.J. et al. (2007) Large-scale mapping and validation of Escherichia coli transcriptional regulation from a compendium of expression profiles. PLoS Biol., 5, 0054-0066.

Huynh-Thu,V.A. et al. (2010) Inferring regulatory networks from expression data using treebased methods. PLoS One, 5, 1-10.

Langfelder,P. and Horvath,S. (2008) WGCNA: an R package for weighted correlation network analysis. BMC Bioinformatics, 9, 559.

Love,M.I. et al. (2014) Moderated estimation of fold change and dispersion for RNA-seq data with DESeq2. Genome Biol., 15, 1-21.

Marbach,D. et al. (2012) Wisdom of crowds for robust gene network inference. Nat. Methods, 9, 796-804.

Margolin,A.A. et al. (2006) ARACNE: An algorithm for the reconstruction of gene regulatory networks in a mammalian cellular context. BMC Bioinformatics, 7, 1-15.

Meyer,P.E. et al. (2008) Minet: A r/bioconductor package for inferring large transcriptional networks using mutual information. BMC Bioinformatics, 9, 1-10.

Morgan,M. et al. (2021) BiocParallel: Bioconductor facilities for parallel evaluation.

Morgan,M. et al. (2020) SummarizedExperiment: SummarizedExperiment container.

Parsana,P. et al. (2019) Addressing confounding artifacts in reconstruction of gene coexpression networks. Genome Biol., 20, 94.

Petereit,J. et al. (2016) petal: Co-expression network modelling in R. BMC Syst. Biol., 10, 51.

Russo,P.S.T. et al. (2018) CEMiTool: a Bioconductor package for performing comprehensive modular co-expression analyses. BMC Bioinformatics, 19, 56.

Shin,J. et al. (2020) A network-based comparative framework to study conservation and divergence of proteomes in plant phylogenies. Nucleic Acids Res., 1-23. 
bioRxiv preprint doi: https://doi.org/10.1101/2021.04.10.439287; this version posted April 11, 2021. The copyright holder for this preprint (which was not certified by peer review) is the author/funder, who has granted bioRxiv a license to display the preprint in perpetuity. It is made available under aCC-BY-NC 4.0 International license. 